\title{
FACTEURS INFLUENÇANT LES PARAMĖTRES DE REPRODUCTION DES BREBIS OULED DJELLAL
}

\author{
FACTORS INFLUENCING REPRODUCTION PARAMETERS \\ OF EWES OULED DJELLAL \\ Arbouche, R. ${ }^{1 A}$; Arbouche, H.S. ${ }^{1 B} ;$ Arbouche, F. ${ }^{1 *}$ et Arbouche, Y. ${ }^{1 C}$ \\ ${ }^{1}$ Laboratoire Agriculture et fonctionnement des écosystèmes. Université d'El Tarf. Algérie. \\ Arafik_arbouche@yahoo.fr; Barbouchehs@yahoo.fr; *arbouchefodil@yahoo.fr; 'yas.arbouche@yahoo.fr;
}

\section{MoTS CLÉS}

Fécondité. Fertilité. Ovin. Prolificité. Système d'élevage.

\section{RÉSUMÉ}

L'étude des paramètres de reproduction des brebis Ouled Djellal $(n=269)$, menée en semiintensif dans un milieu semi- aride, fait ressortir un taux de fertilité de $88 \%$, un taux de fécondité de $95 \%$ et un taux de prolificité de $111 \%$. L'influence des différents facteurs sur les paramètres de reproduction, fait ressortir sur la fertilité, une influence significative $(p<0,05)$ de l'état physiologique lors de la lutte, un effet très significative de l'âge des brebis et un résultat hautement significative $(p<0,001)$ du mode de lutte, du mois de lutte ainsi que de l'interaction état physiologique lors de la lutte $\times$ mode de lutte. Pour la fécondité, on note un effet significatif $(p<0,05)$ de l'âge des brebis, très significatif $(p<0,01)$ de l'état physiologique lors de la lutte et hautement significatif $(p<0,001)$ pour le mode de lutte et le mois de lutte. Quant à la prolificité, elle est influencée significativement $(p<0,05)$ par l'état physiologique lors de la lutte et très significativement $(p<0,001)$ par le mois de lutte.

\section{SUMMARY}

The study of the reproductive parameters of the ewes Ouled Djellal ( $n=269)$, conducted in semiintensive in semi-arid environment, shows a fertility rate of $88 \%$, a fecundity rate of $95 \%$ and a prolificacy rate was $111 \%$. The influence of different factors on the reproduction parameters, highlights on fertility, a significant influence $(p<0.05)$ of the physiological state during the mating, an

\section{AdDitiONAL KEYWORDS}

Fecundity. Fertility. Livestock system. Prolificity. Sheep.

effect very significant of ewes age and a result highly significant $(p<0.001)$ of mode of mating, of month of mating and for the interaction of physiological state during the mating $\times$ mode of mating. For fecundity, there is a significant effect $(p<0.05)$ of the ewes age, very significant $(p<0.01)$ of the physiological state during the mating and highly significant $(p<0.001)$ for mode of mating and the month of mating. As for prolificacy, it is significantly influenced by the physiological state during the mating and very significantly by the month of mating.

\section{INTRODUCTION}

En Algérie, le cheptel ovin représente la plus grande ressource animale, il est estimé à plus de 19 millions de têtes dont 10 millions de brebis reproductrices (MADR, 2006). L'élevage ovin, malgré son importance économique et sociale, est mal conduit, tant en organisation technique qu'en fonctionnement de ses systèmes de production car la majorité des éleveurs mènent leurs troupeaux en reproduction durant la contre saison (juin, juillet et aout) afin de pouvoir bénéficier des repousses de végétation aux premières pluies d'octobre. Les méthodes de contrôle et de maîtrise de la reproduction, permet de choisir la période de mise-bas et d'optimiser 
la taille de la portée (Chemineau et al., 1996). Elles se répartissent en deux catégories, l'une de nature hormonale et l'autre par l'effet bélier. L'induction et la synchronisation des chaleurs chez les brebis par l'utilisation de la PMSG (pregnant mare serum gonadotropin) est fréquemment associée à un traitement préliminaire aux progestagènes basé sur la mise en place d'une éponge vaginale imprégnée de FGA (fluorogestone acetate) (Castonguay, 2006).

\section{MATERIELETMETHODES}

La ferme pilote Yahia Ä̈chouche se situe au niveau de la wilaya de Bordj Bou Arreridj. Sur une période de vingt ans, la température et les précipitations moyennes annuelles sont de $15,3^{\circ} \mathrm{C}$ et $39,9 \mathrm{~mm}$ respectivement.

L'expérimentation s'est effectuée sur un troupeau de 269 brebis, de race ovine Ouled Djellal type Hodna dont le mode de conduite est en semi-intensif avec pâturage durant toute la journée et un complément alimentaire à base d'orge et de produit semi-fini (issues de meunerie) à leur retour le soir en sus de la paille. L'eau étant mise à disposition ad libitum. Ce troupeau est subdivisé en deux lots.

Le premier lot a été mené en reproduction contrôlée (synchronisation des chaleurs) à travers les éponges vaginales imprégnées de $60 \mathrm{mg}$ de FGA (fluorogestone acetate, Pharmplex Pty Ltd) après sevrage pour une durée de 14 jours, la PMSG (pregnant mare serum gonadotropin) (folligon à 1000 UI: lyophylisat injectable + solvant) injecté à 500 UI, avec monte naturelle. Il est constitué de 125 femelles, réparties en 100 brebis allaitantes, 25 non allaitantes, à âge hétérogène variant entre 18 mois et 6 ans.

Le second lot, mené en lutte naturelle sans synchronisation, comprenant 144 femelles dont 116 brebis allaitantes et 28 non allaitantes. L'âge compris entre 18 mois et 6 ans.

Pour les mâles, 15 béliers sélectionnés en provenance du Centre d'Insémination Artificielle de la région d'Ouled Djellal
(Wilaya de Biskra) ont été ramenés et mis à l'écart de la vue des brebis.

La statistique descriptive et l'analyse de variance du model linaire général univarié (ANOVA), ont été effectuées avec le logiciel SPSS (version 18, 2008).

\section{RESULTATSETDISCUSSION}

Les taux globaux de fertilité, fécondité et prolificité sont de $88 \pm 0,02,95 \pm 0,04$ et $111 \pm 0,05 \%$ respectivement. Ils sont supérieurs au taux de l'année 2009 (80, 87 et $108 \%$ ), enregistrés par Bouafia et Lamara (2009) sur le même site.

L'age des brebis est très significative $(\mathrm{p}<0,01)$. Les brebis âgées de 3 ans ont un taux de fertilité plus élevé (97\%) alors que celles de 4 ans ont le taux le plus bas $(77,5 \%)$. Les brebis de 2, 5 et 6 ans ont des taux intermédiaires. Selon Boujenane et Chikhi, (2006), l'âge des brebis a un effet hautement significatif $(\mathrm{p}<0,001)$ sur la fertilité.

L'etat physiologique des brebis lors de la lutte a un effet significatif $(\mathrm{p}<0,05)$ sur la fertilité. Les brebis non allaitantes ont un taux de $94 \%$ nettement supérieur à celui des brebis allaitantes $(64 \%)$. Cette différence est à imputer à la production laitière qui est entretenu grâce aux stimuli de la tétée. Atti et Abdennebi (1995), à travers l'analyse de la fertilité des brebis en fonction des notes d'état corporel (NEC) à la lutte, ont montré que ce paramètre s'améliore d'une classe à l'autre. Cependant, Thomson et Bahhady (1988) et Abdennebi et Khaldi (1991), ont trouvé que les brebis fertiles sont moins lourdes avant la lutte que les brebis stériles.

L' effet du mode de lutte est hautement significatif $(p<0,001)$ sur la fertilité. Le lot synchronisé a eu une performance de $16,8 \%$, ce faible taux est dû à l'introduction de béliers à faible ardeur sexuelle (en majorité des antenais). Lors du retour en chaleur (17 jours après), le taux de fertilité n'est que de $16,3 \%$. Alors qu'au troisième retour, le taux est passé à 75,8\%. Le lot lutte naturelle a enregistré un taux de fertilité de $90 \%$. Pendant 
le premier retour des chaleurs, les béliers étaient performants, et le faible taux de fertilité enregistré semble être dû à l'existence au sein de l'organisme des brebis d'un taux d'hormones résiduelles. Lorsque l'effet des hormones injectées s'estompe, elles induisant un équilibre hormonal des brebis et un taux de fertilité de 75,8\%. Selon Harkat et Lafri (2007), le taux de fertilité n'augmente pas avec la quantité de PMSG employée (60, 75 et $60 \%$ pour des quantités de 400 UI, 500 UI et 600 UI respectivement), alors que pour le lot témoin, le taux de fertilité était de $60 \%$.

Le mois de lutte est hautement significative $(p<0,001)$ sur la fertilité. Le taux maximal est à attribuer aux mois de mai et juillet (69,4 et $69 \%$ respectivement), alors que les taux les plus bas sont signalés aux mois d'avril et août (53,2 et $51 \%$ respectivement). Merghem (2008), a constaté que le taux de fertilité les plus élevé a été enregistré aux mois d'avril et mai avec $83 \%$ pour la même race.

L'interaction etat physiologique lors de la lutte $\times$ mode de lutte est hautement significative $(p<0,001)$ et découle de l'influence respective de ces deux paramètres sur la fertilité. Quoique l'état physiologique ne soit que significatif $(p<0,05)$, sa combinaison avec le mode de lutte $(\mathrm{p}<0,001)$, les rend simultanément hautement significatifs $(\mathrm{p}<0,001)$, d'où l'effet de la synchronisation.

L'age des brebis a un effet significatif $(\mathrm{p}<0,05)$ sur la fécondité. Les brebis âgées de 3 ans ont un taux élevé de fécondité (112,5 $\%)$, le taux le plus faible est à attribuer aux brebis âgées de 6 ans (83,3\%); alors que les brebis âgées de 2,4 et 5 ans ont une fécondité de 91,$4 ; 85$ et $96,4 \%$ respectivement.

L'etat physiologique des brebis lors de la lutte a une influence très significative $(\mathrm{p}<0,01)$ sur la fécondité. Une différence de fécondité de 33,2\% est à noter entre les brebis non allaitantes (107\%) et les allaitantes (73,8\%). Cappai et al. (1984), ont mis en évidence que le niveau de production laitière au moment de la saillie a une influence sur la fécondité.
Le mode de lutte est hautement significatif $(p<0,001)$. Le taux de fécondité pour le lot synchronisation est faible $(18,8 \%)$, il est à mettre au compte de la faible performance des reproducteurs. Lors du retour en chaleur (17 jours après), le taux de fécondité n'est que de 18,3\%. Au second service, le taux est passé à $106 \%$ après élimination des résidus hormonaux pré injectés. Le lot lutte naturelle a enregistré un taux de fécondité de $122 \%$. Bouafia et Lamara, (2009), au niveau du même site d'étude, ont signalé que le taux de fécondité du lot synchronisé (124\%), était supérieur de $47 \%$ par rapport au lot mis en lutte naturelle (77\%).

Le mois de lutte a une influence hautement significative $(\mathrm{p}<0,001)$ sur la fécondité. Le taux maximal est atteint au mois de mai (118,7\%), alors que le taux le plus faible au mois d'août (50\%). Pour les mois d'avril et juillet, les taux sont de 64 et $75 \%$ respectivement. La faible performance du mois d'avril (saison sexuelle) est la résultante de la conduite d'élevage où les brebis durant ce mois ont été sous alimentées (11 g/jour/brebis d'orge avec une ration de base constituée de paille) et aux mois de juillet et août, l'alimentation s'est résumée au pâturage des chaumes. Selon O'brien, (2002), une brebis qui bénéficie d'une alimentation de bonne qualité durant les 2 à 3 mois qui précédent la lutte, est une garantie de meilleures performances de reproduction.

L'age des brebis n'influence pas la prolificité $(p>0,05)$. Cette observation est avancée par Dekhili (2004), mais contredite par Dekhili (2002). Selon cet auteur, l'effet du milieu est mis en cause.

L'etat physiologique des brebis lors de la lutte est significatif sur la prolificité $(\mathrm{p}<0,05)$, nous remarquons que le taux de prolificité est de $114,8 \%$ pour les brebis non allaitantes alors que le taux des brebis allaitantes est de $108 \%$. Les brebis, mères de doublés utilisent davantage leurs réserves corporelles au cours de la gestation et de l'allaitement que les mères de sim- 


\section{ARBOUCHE, ARBOUCHE, ARBOUCHE ET ARBOUCHE}

ples (Gunn et al., 1969).

Le mois de lutte a un effet très significatif $(\mathrm{p}<0,01)$ sur la prolificité. Les brebis luttées durant le mois d'avril (130\%) et mai (129\%) sont plus performantes que celles luttées durant le mois de juillet $(118 \%)$ et août $(100 \%)$. Ce résultat est à attribuer à la complémentation alimentaire basée principalement sur l'orge.

\section{BIBLIOGRAPHIE}

Abdennebi, L. et Khaldi, G. 1991. Performances de reproduction d'un troupeau ovin prolifique de race Barbarine. Ann INRAT, 12: 64-30.

Atti, N. et Abdennebi, L. 1995. Etat corporel et performance de la race ovine Barbarine. Centre International des Hautes Etudes Méditerranéen. Options Méditerranéennes, 6: 75-80.

Bouafia, I. et Lamara, A. 2009. Analyse des performances de reproduction et de productivité de la brebis Ouled Djellal dans la ferme Ben Aïchouche à Bordj Bou Arerridj Mémoire d'ingénieur agronome. Université de Sétif. 86 pp.

Boujenane, I. et Chikhi, A. 2006. Paramètres génétiques et phénotypiques des performances de reproduction des brebis des races Boujaâd et Sardi au Maroc. Revue d'Élevage Vétérinaire Pays Tropicaux, 59: 51-57.

Cappai, P.; Cognie, Y. and Branca, A. 1984. Use of the male effect to induce sexual activity in Sarda ewes. In: The male in farm animal reproduction. Ed. M. Courot. Martinus Nijhoff Publishers. The Hague. pp. 316-323.

Castonguay, F. 2006. Techniques d'induction des chaleurs. L'éponge vaginale. Fiche technique groupe de recherche sur les ovins. Agriculture et agroalimentaire. Canada. http://www.ovins. fsaa.ulaval.ca/uploads/tx_centrerecherche/ FICHE_TECH_EPONGES.pdf (20/06/2012).

Chemineau, P.; Cognie, Y. et Heyman, Y. 1996 Maitrise de la reproduction des mammifères d'élevage. Institut National de Recherches

\section{CONCLUSION}

Au niveau de la wilaya de Bordj Bou Arreridj, les paramètres de reproduction sont influencés par les facteurs suivants: l'âge de la brebis; l'état physiologique des brebis lors de la lutte; le mode et le mois de lutte et l'interaction état physiologique des brebis lors de la lutte $\times$ le mode de lutte.

Agronomiques. Production Animale (hors série): 5-15.

Dekhili, M. 2002. Performances reproductives des brebis Ouled Djellal nées simples et doubles. gème. Rencontre Recherche Ruminant. 155 pp.

Dekhili, M. 2004. Étude de la productivité d'un troupeau de brebis de race Ouled Djellal. 11 ème Rencontre Recherche Ruminant. $151 \mathrm{pp}$.

Gunn, R.G.; Doney, J.R. and Russel, A.J.F. 1969. Fertility in Scottish Blackface ewes as influenced by nutrition and body condition at mating. $J$ Agr Sci, 73: 289-294.

Harkat, S. et Lafri, M. 2007. Effet des traitements hormonaux sur les paramètres de reproduction chez les brebis Ouled Djellal. Courrier du Savoir $N^{\circ}$ 08. Juin 2007. pp. 125-132.

MADR. 2006. Statistiques agricoles. Ministère de l'agriculture et du développement rural. Algérie. $64 \mathrm{pp}$.

Merghem, M. 2008. Caractérisations des paramètres zootechniques des ovins dans la région de Sétif. Mémoire de Magister en agriculture et développement durable. Université de Sétif. 62 pp.

O'brien, A. 2002. Flushing the ewe flock: is it beneficial? Factsheet animal science Ministry of Agriculture and Food Ontario. 56 pp.

Thomson, E.F. and Bahhady, F.A. 1988. Note of the effect of live weight at mating on fertility of Awassi in semi-arid north west Syria. Anim Prod, 47: 505-508. 